\title{
Double Coil-Less Fluxgate in Bridge Configuration
}

\author{
M. Butta ${ }^{1}$, P. Ripka ${ }^{1}$, Joaquín Pérez Navarrete ${ }^{1,2}$, and M. Vázquez ${ }^{3}$ \\ ${ }^{1}$ Faculty of Electrical Engineering, Czech Technical University in Prague, Praha EU 16627, Czech Republic \\ ${ }^{2}$ Universidad Politécnica de Valencia, ETSIT, Valencia 46022, Spain \\ ${ }^{3}$ Institute for Materials Science of Madrid, CSIC, Madrid 28049, Spain
}

\begin{abstract}
In this paper, a new method for excitation of coil-less fluxgate is presented. The purpose of this method is to reduce the spurious component of the output voltage, allowing us to increase the amplification. The method is based on the employment of two coil-less fluxgates in a double bridge, which injects pulsing current in opposite direction in each wire. By taking the difference of the voltages on the two wires, we suppress the component of the voltages, which does not change under application of external measured field. The sensitive axes are in opposite direction, so the wire feels opposite field. As a result, we will obtain an output voltage with low peak value, including only the component of the voltage that changes when we apply external field. Finally, we propose an improved version of the double bridge to allow the employment of two sensing elements with difference characteristics. This is obtained by optimizing the suppression of the spurious voltages and, at the same time, setting independently chosen values of exciting current for each wire.
\end{abstract}

Index Terms-Bridge, coil-less, double, fluxgate, orthogonal, pulse excitation.

\section{INTRODUCTION}

$\mathbf{F}$ LUXGATE sensors are usually classified in two categories: parallel and orthogonal ones. The mainstream of research and applications is focused on parallel fluxgates because of their better properties. However, orthogonal fluxgates have been recently rediscovered thanks to the employment of magnetic microwire as core, instead of bulk cylinders.

One of the advantages of the orthogonal fluxgate mode is the absence of any output at pickup coil in case of no external field measured, which allows us to increase the gain of the amplification. On the other hand, the amplification of output voltage in single-core parallel-type fluxgate is limited by the peak value of the voltage, which is present even without external field.

Coil-less fluxgate is a special type of orthogonal fluxgate. The sensor is composed of a single magnetic microwire excited by the current flowing through it. The output voltage is taken on the terminations of the wire itself, without need of any coil [1]. Even if such a structure reminds other types of magnetic sensors, it has been shown that it works in fluxgate mode. The output of coilless fluxgate sensors is composed of an idle resistive component and a magnetic component that shifts under the application of external magnetic field.

Coil-less fluxgate has simple structure (only one wire without coils), but contrary to traditional orthogonal fluxgate, it has nonzero output voltage for zero external field. In fact, it is defined as orthogonal fluxgate because of the orthogonality between the excitation field and the sensing direction. However, its output is characterized by a high spurious component, as typically found in parallel fluxgates. We define the spurious component of the output voltage as that part of the voltage that does not change when magnetic field is applied to the sensor. For instance, a spurious component is the resistive voltage drop

Manuscript received June 20, 2009; revised September 13, 2009 and September 16, 2009; accepted September 16, 2009. Current version published January 20, 2010. Corresponding author: M. Butta (e-mail: buttam1@fel.cvut.cz).

Color versions of one or more of the figures in this paper are available online at http://ieeexplore.ieee.org.

Digital Object Identifier 10.1109/TMAG.2009.2033339 on the wire, which does not depend on magnetic field. Such a component of the voltage increases the maximum value of the voltage, resulting as a limit to maximum amplification, without giving any information about the measured field.

In this paper, we propose a technique that allows us to overcome the problem of such spurious components by using two sensing elements in a double-bridge configuration. In this way, we can take advantage of the simple structure of coil-less fluxgate, with much lower limitation on the amplification gain.

\section{SEnsing ElEment AND Signal Extraction}

For this research, we used magnetic microwires produced in cooperation with the team led by M. Vazquez at the Institute for Materials Science of Madrid, CSIC, Madrid, Spain [2].

The microwires are composed of a glass-coated copper core (40 $\mu \mathrm{m}$ diameter). On the glass coating, we sputter a few nanometers thick layer of gold, and finally we perform electrodeposition of $8-\mu \mathrm{m}$ permalloy layer on it.

The ac excitation current is injected into the copper core, creating a circumferential field that saturates the Permalloy layer in circumferential direction. The voltage measured at the terminations of the wire is composed of two components. The first is the resistive component, i.e., the voltage drop on the copper wire resistance caused by the excitation current. The second component is the inductive component, following the saturation process of the permalloy layer; in this case, we can consider a single-turn coil composed of the wire itself and the return wire, including the circumferential cross section of the permalloy wire as magnetic area. As shown in [1], in case of helical anisotropy, the induction component of the output voltage shifts in time with applied dc measured field.

Pulse excitation has been proven to be suitable for this kind of sensor because is allows strong reduction of power consumption (down to tens of microwatts). Signal extraction is performed by integrating the output voltage for a short time interval on both positive and negative peak [3]. Even if the sum of the integrals on positive and negative peak is null for no applied field, the integrated voltage still has a high peak, which limits the maximum amplification factor. 


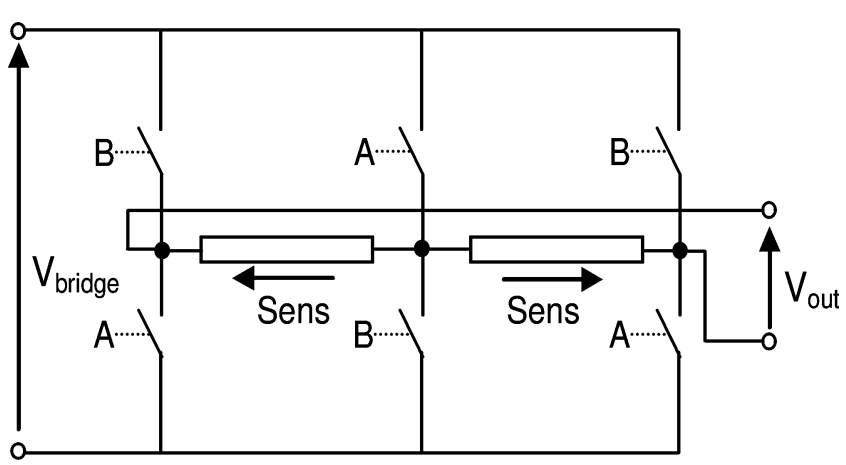

Fig. 1. Double-bridge configuration for simultaneous excitation of two cores of a double coil-less fluxgate.

\section{DOUBLE-Bridge CONFIGURATION}

We propose a simple idea to reduce the peak amplitude of coil-less fluxgate output voltage, and straightforward increase the amplification gain, without degrading the sensor performance. The system is based on two sensing elements connected in a double bridge, as shown in Fig. 1.

The bridge has been realized by IRF7103 Mosfet switches controlled by TPS 28225 drivers. The control signals have been generated by PIC16F737 microcontroller and logic network.

By closing A switches, we inject a current into the wires, and when we close B switches, we inject negative current. In both cases, the two wires are always excited by current flowing in opposite direction.

If we measure the voltage $\mathrm{V}_{\text {out }}$ at the external connection points of the bridge, as shown in Fig. 1, the resistive component of the voltage is suppressed. Indeed at $\mathrm{V}_{\text {out }}$, we have $\mathrm{R}_{1} \cdot \mathrm{I}_{1}+$ $\mathrm{R}_{2} \cdot\left(-\mathrm{I}_{2}\right)=\mathrm{R}_{1} \cdot \mathrm{I}_{1}-\mathrm{R}_{2} \cdot \mathrm{I}_{2}$, which is zero in case of wires with equal resistivity. In this way, we get rid of the resistive component of the output voltage.

However, this configuration would be useless if the sensing direction of the two wires were the same. Indeed, beside the resistive components, we would also cancel the inductive component of the voltage, returning a zero output signal. One could think to use wires with different helical anisotropy angles, and therefore different sensitivity. In this way, we would have as a result an output voltage having sensitivity equal to the difference of the sensitivities of the two wires. Nevertheless, we would have in any case a decrease of the performances (lower sensitivity), and we should take care about always using wires with different sensitivity.

A smarter solution is to use two wires with sensing axes pointing in opposite directions. On the output voltage, we have the sum of the inductive peaks due to the magnetization of both wires. Let us consider the positive peak: If the wire is sensing a positive external field, the peak will shift to the left (that is, saturation occurs for lower excitation field). The second wire, with opposite sensing direction, will sense a negative field; therefore, its peak will move to the right. If we consider the difference of such peaks, we find out that the resulting voltage is null for zero measured field (both peaks are in the same position), and two small knolls rise when we apply external magnetic field (peaks are moving in different directions).

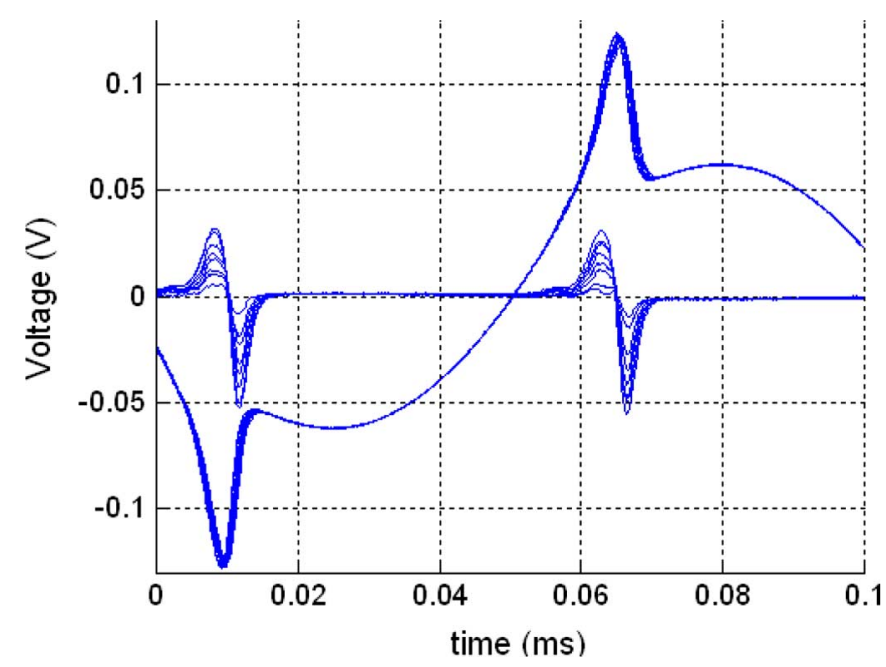

Fig. 2. Output voltage for double wire configuration, simulated for sine-wave excitation, and external field from 0 to $40 \mathrm{~A} / \mathrm{m}$ (5-A/m steps). The original voltage on the wire's termination is also shown. We can observe that the peak value of output voltage of the bridge is much lower than original voltage.

If we consider the negative peak, the situation is simply reversed. The first wire will have an inductive component in the form of a peak shifting to the right when we apply positive external field, whereas the peak of the second wire will shift left. As the sign of the peak is now reversed, the difference results in the same knolls we obtained for positive peak.

Finally, we achieve an output voltage $V_{\text {out }}$, where the resistive voltage is suppressed and the inductive component does not shift to the left or to the right anymore, but gives rise to small knolls.

\section{Simulation}

We first performed simulation of such principle using sinewave excitation rather than pulsing excitation. The goal was to verify this idea for the simple case of single frequency.

We have digitized the output voltage of a single wire $V_{\text {wire }}$, applying different values of external field by means of a Helmholtz coil $(-160 \div 160 \mathrm{~A} / \mathrm{m}$ in steps of $20 \mathrm{~A} / \mathrm{m})$. We have computed the waveform we would get if we had two identical wires, and we arranged them in such a way that they are excited by opposite current and with sensing axes in opposite directions. That is, the resulting output at $40 \mathrm{~A} / \mathrm{m}$ is obtained

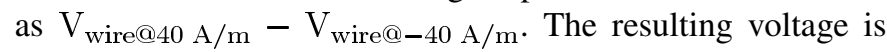
shown in Fig. 2 in the case of positive external field and in Fig. 3 in the case of negative field. In Fig. 2, the original voltage $\mathrm{V}_{\text {wire }}$ on the terminations of the wires is also shown to let the reader evaluate the reduction of peak amplitude obtained by this method.

We have performed digital extraction of second harmonic: The sensitivity for the original voltage of the wire $V_{\text {wire }}$ turned out to be $11.6 \mathrm{~V} / \mathrm{T}$, while the sensitivity of the output signal obtained by simulating two identical wires working on the proposed mode was $23.2 \mathrm{~V} / \mathrm{T}$ - that is two times the original sensitivity. This is clearly explained by the fact that we are subtracting two characteristics with opposite sign sensitivity, and finally we get the sum of them. 


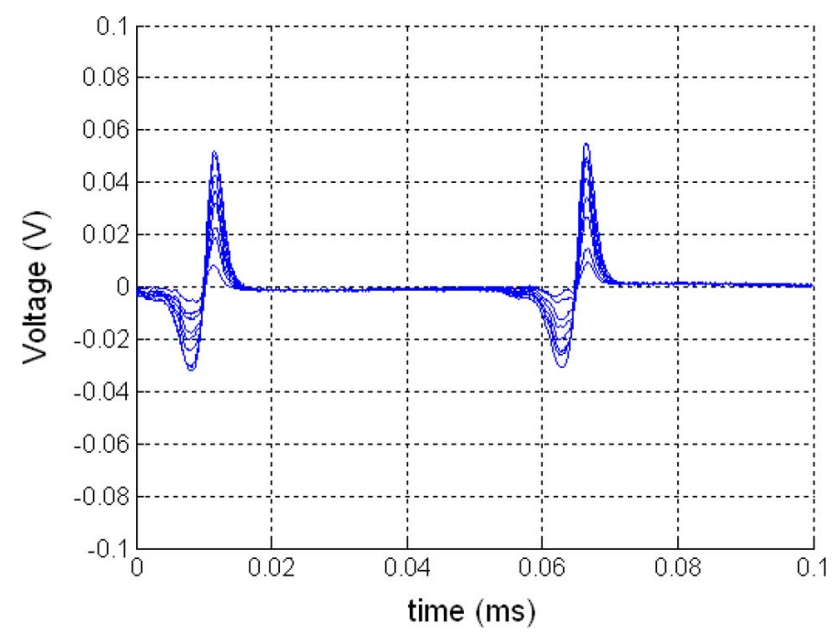

Fig. 3. Output voltage for double-wire configuration, simulated for sine-wave excitation, and external field from 0 to $-40 \mathrm{~A} / \mathrm{m}$ (5-A/m steps).

\section{Measurement With Different Wires}

We have proved that in the ideal case of equal wires, the method gives lower spurious output voltage and double sensitivity. Now, we want to check if the results still hold in the real case of pulse excitation and especially in the case of two wires with different characteristics.

In fact, methods employing two magnetic sensing elements have already been proposed for other types of sensors [4]. Unfortunately, such methods have proven to be not very successful. The main goal was to obtain a linear characteristic out of a nonlinear sensor by compensating the nonlinearity of the first sensor with opposite nonlinearity of the second sensor. This idea is theoretically fine, but it fails for practical applications because it is not very easy to obtain magnetic wires with identical characteristics. In some cases, even a small difference in the characteristic of the wire can determine a complete loss of any improvement.

However, in our case, we already have two linear elements, and we sum their characteristics (we subtract the signals with opposite sensing directions). As the sum is a linear operator, we should have as a result the sum of two linear characteristics, which is supposed to be linear as well.

We excited the wires by the double bridge using $40 \mathrm{~mA}$ peak current at $10 \mathrm{kHz}$. The output signal has been digitized by the first channel on a NI- PXIe-5122 acquisition board (14 bit, $100 \mathrm{MHz}$ ). The control unit that generates the signals for the bridge's switches also generates the gating signal for integration. We measured such signal on the second channel of the acquisition board. Finally, we numerically multiply the output voltage times the gating signal, and we perform numerical integration of the resulting waveform. Fig. 4 shows the measured voltage $\mathrm{V}_{\text {out }}$ for external field varying from 0 to $50 \mathrm{~A} / \mathrm{m}$ in steps of $10 \mathrm{~A} / \mathrm{m}$. The integrating gate is shown, too. Together with the output voltage of the bridge $\mathrm{V}_{\text {out }}$, the voltage of one wire is shown. The peak amplitude of the spurious voltage obtained with this method is more than five times lower than the original voltage.

The output characteristic has been obtained by the digital integration of the gated output voltage. Fig. 5 shows the resulting characteristic together with the characteristic obtained using the

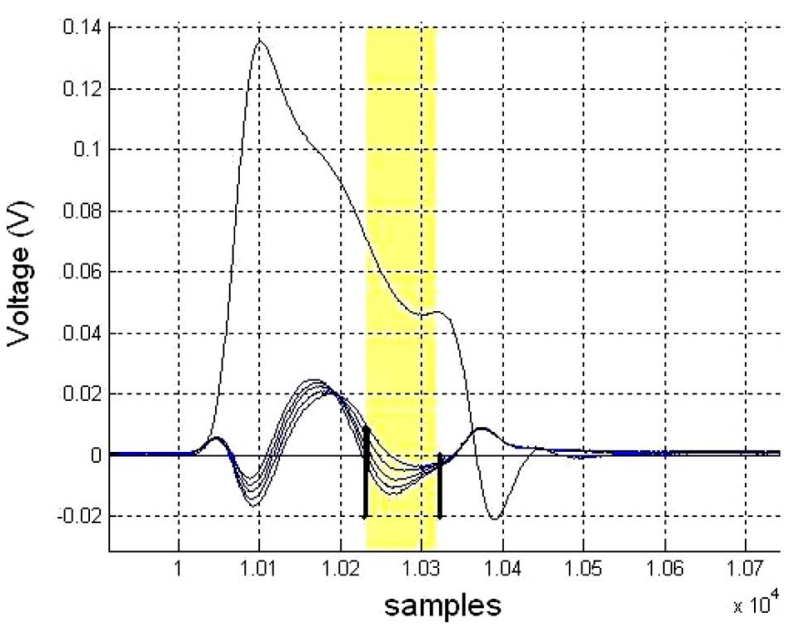

Fig. 4. Output voltage of double bridge with two pulse-excited coil-less fluxgates, measured for external field from 0 to $50 \mathrm{~A} / \mathrm{m}$ (10-A/m steps). For the sake of clarity, only the positive pulse is shown. The gating window is highlighted. The voltage on one wire is also shown.

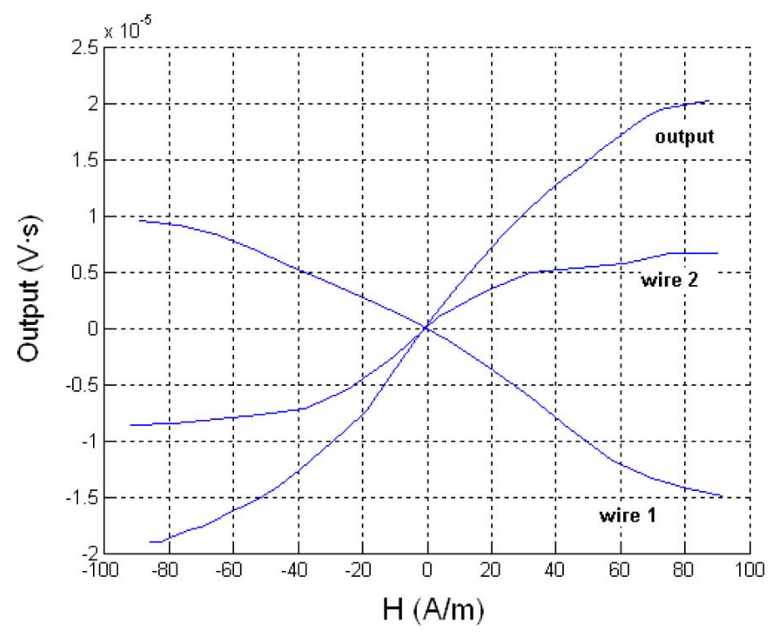

Fig. 5. Output characteristic measured on both wires composing the bridge and on the total output of the bridge. The total output characteristic corresponds to the sum of the characteristics measured on the single wires.

same gating signal on the two sensors composing the bridge. We can clearly see that the output characteristic is the difference of the two wires' characteristics, as theoretically derived. The sensitivity of the first wire is $-0.182 \mathrm{~V} \cdot \mathrm{s} / \mathrm{T}$, the sensitivity of the second wire is $0.177 \mathrm{~V} \cdot \mathrm{s} / \mathrm{T}$, and the output sensitivity is $0.36 \mathrm{~V} \cdot \mathrm{s} / \mathrm{T}$, that is, the sum of them.

\section{MODIFIED BRIDGE}

Generally, two different wires have different impedance. Therefore, it is rather unlikely they will be excited by the same current when used in the bridge configuration shown in Fig. 1.

Moreover, we should consider that the sensitivity of the wire depends on the excitation current amplitude. From one side, we would prefer similar excitation current for both wires in order to better suppress spurious voltage component. On the other end, similar current does not necessarily imply similar characteristic.

Therefore, we have developed a modified bridge, which allows us to both regulate the current in order to obtain similar characteristics of the two wires and simultaneously to regulate 


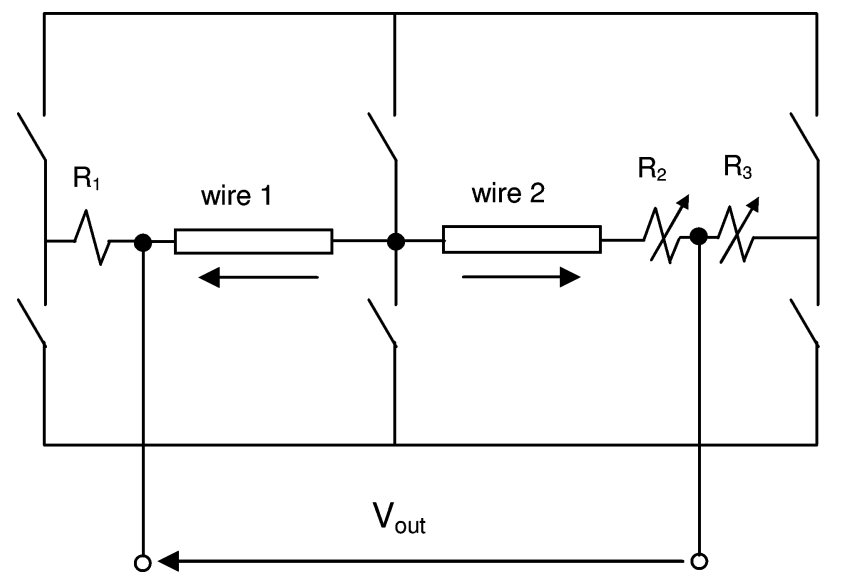

Fig. 6. Improved version of the excitation bridge. This version is suitable in the general case when wires have different properties in order to tune proper current in each wire and erase most spurious voltage at the same time.

the matching of the two voltages to get the best suppression of spurious voltages. The scheme is shown in Fig. 6: We add a resistor $R_{1}$ in series to wire 1 and two variable resistors $R_{2}$ and $R_{3}$ in series to wire 2 . We regulate the value of $R_{2}$ and $R_{3}$ so that the total resistance on the right side and the total resistance on the left side determine a combination such that proper current flows in wire1 and wire2. These two currents are not necessarily equal: Their amplitude must be such that the resulting characteristics of the wires are similar (at least they should be both well saturated, and current should be not too high-a condition that could be achieved for different current amplitudes, according to wire parameters). Then, we regulate the amplitude of $\mathrm{R}_{2}$ in order to have similar voltage amplitude on the left and right side of the bridge; in this way, we get the best suppression of spurious voltage. While changing the value of $R_{2}$, we simultaneously change the value of $R_{3}$, so that the sum $R_{2}+R_{3}$ does not change, in order to not modify the value of the currents.

In this case, wire 2 is supposed to have lower resistance than wire $1 ; \mathrm{R}_{2}$ compensates the difference. However, in case we do not know the resistance of the wires, we can add another resistor in series to $R_{1}$ in order to be able to match the voltages, also in case the resistance of wire 2 has bigger resistance than wire 1 , without modifying the connections.
Based on the principle shown in Fig. 6, we could achieve the same result using a potentiometer, instead of $R_{2}$ and $R_{3}$, in order to modify the match of the right-side and left-side voltage by simply acting on a single knob and adding a variable resistor in series for the previous setting of the total resistance on the right side (to have proper current values).

\section{CONCLUSION}

In this paper, we have shown a method for the excitation of coil-less fluxgates. We employed a double bridge to inject current with opposite signs into two sensing elements. The output voltage is the difference of the voltages on the wires: The spurious voltage is suppressed, and only the changing part of the voltage is considered. The peak amplitude of the output voltage is finally reduced, allowing us to increase the gain of the input amplifier.

We have shown how this method is not affected by using magnetic microwires with different parameters, as the resulting output characteristic is simply the sum of the single characteristics of the wires.

Finally, we propose a modified version of the excitation bridge, which allows us to set the proper current amplitude in each wire and, at the same time, to totally suppress the spurious voltage in the output.

\section{REFERENCES}

[1] M. Butta, P. Ripka, S. Atalay, F. E. Atalay, and X. P. Li, "Fluxgate effect in twisted magnetic wire," J. Magn. Magn. Mater., DOI: 10.1016/j. jmmm.2008.04.176.

[2] M. Butta, P. Ripka, G. Infante, G. A. Badini-Confalonieri, and M Vázquez, "Bi-metallic magnetic wire with insulating layer as core for orthogonal fluxgate," IEEE Trans. Magn., vol. 45, no. 10, pp. 4443-4446, Oct. 2009.

[3] M. Butta and P. Ripka, "Pulse excitation of coil-less fluxgate," in Proc. IEEE Sensors 2008 Conf., Lecce, Oct. 26-29, 2008, pp. 379-382.

[4] M. Malátek, P. Ripka, and L. Kraus, "Double-core GMI current sensor," IEEE Trans. Magn., vol. 41, no. 10, pp. 3703-3705, Oct. 2005.

[5] Magnetic Sensors and Magnetometers, P. Ripka, Ed. Norwood, MA: Artech House, 2001, pp. 78-79.

[6] F. Primdahl, "The fluxgate mechanism, Part I: The gating curves of parallel and orthogonal fluxgates," IEEE Trans. Magn., vol. MAG-6, no. 2, pp. 376-383, Jun. 1970. 